\section{$\angle$ Research Square}

Preprints are preliminary reports that have not undergone peer review.

They should not be considered conclusive, used to inform clinical practice, or referenced by the media as validated information.

\title{
Mechanism of Astragalus Membranaceus in the Treatment of Diabetic Nephropathy Based on Network Pharmacology
}

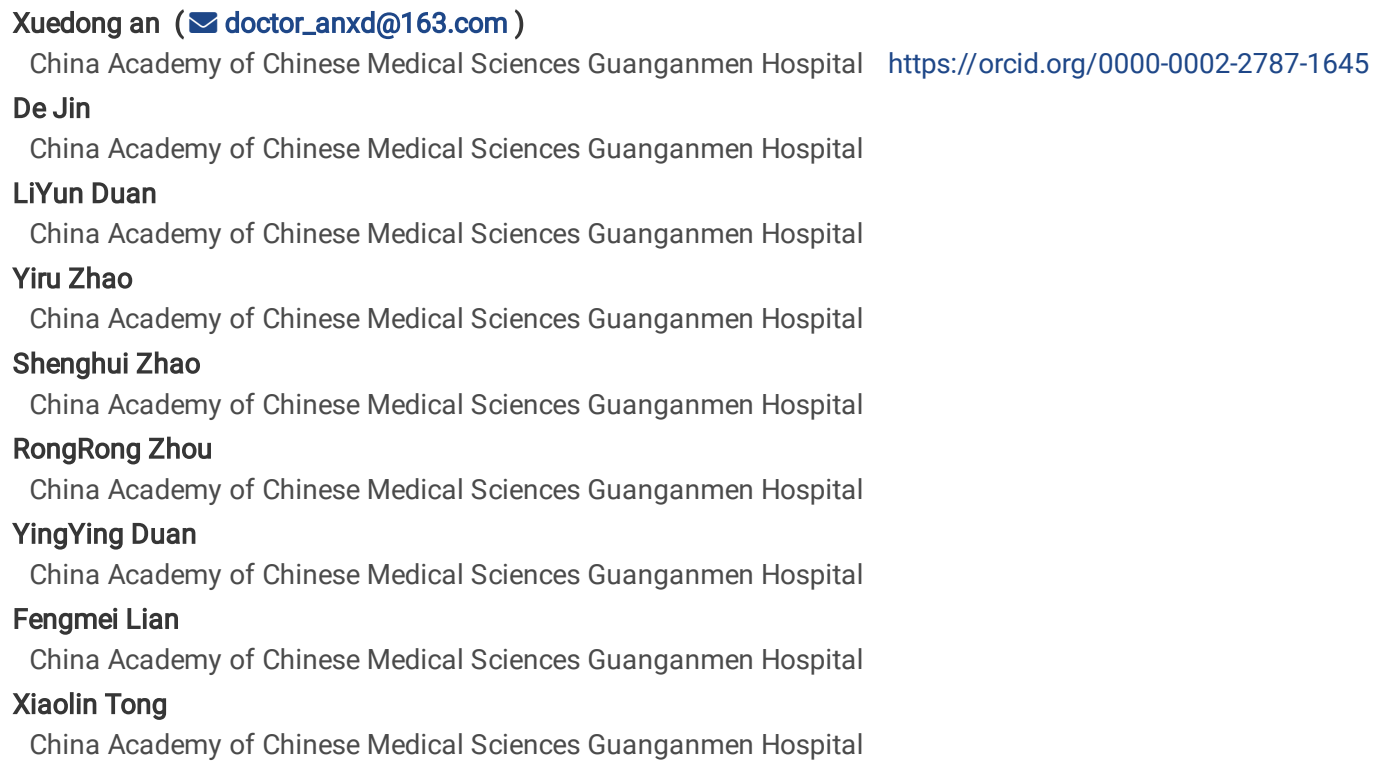




\section{Abstract}

Background: Diabetic nephropathy (DN), which affects more than $40 \%$ of diabetic patients, is still the main cause of end-stage renal disease in most countries. Prescription containing Astragalus Membranaceus (AM) in the treatment of early stage DN with significant effect, but the mechanism is unclear, which we would explore based on network pharmacology.

Method: First, we searched the database of China National Knowledge Internet, Wanfang Database, Chinese Biomedical Literature Database, PubMed, EMBASE, Cochrane database about the randomized, single (double) blind, controlled clinical studies of prescription containing AM in the treatment of DN, determined the effectiveness of prescription containing AM in the treatment of DN. Then, the effective components of AM in Traditional Chinese Medicine systems taxonomy database and analysis platform (TCMSP), Traditional Chinese medicine integrated database (TCMID) and Bioinformatics analysis tool for molecular mechanism of traditio Internal Chinese medicine (BATMAN-TCM) database were searched. According to the oral utilization $\geq 30 \%$ and drug-like $\geq 0.18$, the effective components were screened. PubChem and health information technology (HIT) databases were searched for query validation targets and Simplified molecular input line entry specification (SMILES) of the effective ingredients, and Swisstarget prediction database was used to obtain prediction targets (possibility >0). Drugbank, transient triple differential (TTD), and DisGeNET were searched for the targets related to DN. Finally, the data were integrated by the Cytoscape software, the drug-target-disease network was drawn, the protein interaction network was drawn with String database, and the signal pathway enrichment analysis was carried out with ClueGO.

Result: The results showed that prescription containing AM could effectively reduce the urinary protein excretion rate [95\% md-43.30 (- $57.00,-29.61)] .51$ active components, 396 verified targets and 2330 predicted targets were searched. A total of 120 DN related targets were retrieved. 21 targets were found with Cytoscape. The main pathways were Interleukin-4 and 13 signaling, Activation of Matrix Metalloproteinases, EPH-Ephrin signaling.

Conclusion: Prescription containing AM could effectively reduce the urinary protein excretion rate of DN patients. We speculated that AM mainly treated DN by regulating Angiotensin-converting enzyme (ACE), Vascular endothelial growth factor A (VEGFA), Janus Kinase 1 (JAK1), and interleukin-4 (IL-4) and interleukin13 (IL-13) signaling, activation of matrix metalloproteins, ephrin signaling and other signaling pathways based on network pharmacology, which would be verified by animal experiments or in vitro experiments in the later.

\section{Background}

According to the data provided by International Diabetes Federation, the number of diabetic patients in the world has reached 415 million, and by 2040 , the total number will exceed 600 million. Compared with simple hyperglycemia, diabetic complications would bring more economic and social burden. Among them, diabetic nephropathy (DN), which affects more than $40 \%$ of diabetic patients, is still the main cause of end-stage renal disease in most countries (1, 2). Chronic hyperglycemia, hyperinsulinemia, insulin resistance and hyperlipidemia were related to the development of DN. It may be due to the disorder of glucose and lipid metabolism that causes the activation of some cell events and signal pathways, involving the interaction of hemodynamics and metabolic factors, thus promoting the structural and functional changes of kidney (3). The main histopathological features of DN include hyperplasia and hypertrophy of glomerular cells, thickening of glomerular basement membrane, mesangial expansion and interstitial fibrosis of renal tubules caused by accumulation of extracellular matrix proteins, such as collagen and fibronectin, podocyte loss and podocyte disappearance $(1,4)$. There are evidences showed that the damage of DN podocytes was caused by mechanical stress, inflammatory response, oxidative stress, transforming growth factor- $\beta$ (TGF- $\beta$ ) induction, ReninAngiotensin-Aldosterone system communication and advanced glycation end product accumulation (4). In addition, dimers in a variety of renal signaling pathways, such as adenosine monophosphate activated protein kinase, were associated with the progression of DN and proteinuria (5). In short, we have a preliminary understanding of DN. At present, the treatment of DN includes primary prevention, improved blood glucose, blood pressure, blood lipid, or with symptomatic treatment, such as anti-inflammatory (such as phosphodiesterase inhibitors), renin inhibitory drugs (aliskiren), vitamin D supplement (6-9). However, the current status of treatment is still insufficient, so it is urgent to replace drugs to treat DN.

We found that the application of prescription containing Astragalus Membranaceus (AM) (such as Danggui Buxue Decoction) in the treatment of early stage DN with significant effect, and reusing AM 30-90 g could significantly reduce urinary protein(10). A multicenter prospective cohort study indicated that AM was the most frequently used in the treatment of DN (11). A systematic review preliminarily confirmed the therapeutic effect of AM injection on DN patients, such as reducing urinary protein and improving renal function (12). A pharmacological study had shown that AM could reduce proteinuria, reverse glomerular filtration and improve early or other stages of DN in streptozotocin induced diabetic animal models (13-15). The effect may be related to the effect of AM on TGF- $\beta$ / drosophila mothers against decapentaplegic protein (SMADs) signal pathway, down regulating the expression of TGF- $\beta 1$, SMAD2/3, and delaying the process of renal fibrosis in diabetic mice (16). According to the current evidence, AM may be an alternative drug for DN, but the mechanism is not clear.

Network pharmacology integrates the technology and content of system biology, multi-directional pharmacology, computational biology, network analysis and other disciplines, constructs the multi-level network of "disease-phenotype-gene-drug", and explores the relationship between drugs and diseases from the overall perspective (17). Therefore, we would explore the potential mechanism of AM in the treatment of DN based on network pharmacology. (Fig. 1)

\section{Methods}

\subsection{Treatment of DN with Prescription containing AM}

Randomized, single (double) blind, controlled high-quality studies of treat DN with prescription containing AM were search in China National Knowledge Internet, Wanfang Database, Chinese Biomedical Literature Database, PubMed, EMBASE and Cochrane databases. Search terms were "Astragalus membranaceus or Huangqi" and "diabetic nephropathy or diabetic kidney disease" and "randomized controlled trial ublication Type] OR randomized [Title/Abstract] OR placebo [Title/Abstract]". The deadline was February 1, 2020. Prescription containing AM was mainly aimed at the early treatment of DN, 
according to the Mogensen diagnostic criteria of DN, the urinary protein excretion rate was selected as the main data for analysis to determine the effectiveness of prescription containing $A M$ in the treatment of DN.

1.1 Searching and collecting of active components of AM

Traditional Chinese Medicine systems taxonomy database and analysis platform (TCMSP) (http://lsp.nwsuaf.edu.cn/tcmsp.php, version: 2.3) (18), Traditional Chinese medicine integrated database (TCMID) (http://www.megabionet.org/tcmid/)(19), and Bioinformatics analysis tool for molecular mechanism of tradition Internal Chinese medicine (BATMAN-TCM) (http://bionet.ncpsb.org/batman-tcm/)(20) were used to search active components of AM. The key word of search was "Astragalus Membranaceus or huangqi". According to the characteristics of pharmacokinetics, the active ingredients were searched with the oral availability $\geq 30 \%$ and the drug like $\geq 0.18$.

1.2 Acquisition of verified targets and Simplified molecular input line entry specification (SMILES) of active components of AM

PubChem (https://pubchem.ncbi.nlm.nih.gov/, 2019 Jan 8) (21)and health information technology (HIT) database (http://lifecenter.biosino.org/hit/)(22) were used to obtain the validation targets of active ingredients of AM, and the SMILES of active ingredients from PubChem database.

1.3 Predicted targets of effective components of AM

Swisstarget prediction (http://www.swisstargetprediction.ch) (23) was used to predict drug targets based on ligand structure, which simulated the binding of receptor and ligand by computer and predict their affinity. Firstly, the SMILES of the effective components of AM was collected from PubChem database. For components that cannot get the SMILES directly, ALOGPS (http://www.vccllab.org/lab/logips/, version: 2.1) was used to calculate(24). In the Swisstarget prediction database, we selected the species "Homo sapiens", then inputed the smile structure to save the output file as a CSV format file.

1.4 Acquisition and Collection of DN targets

Taking "diabetic nephropathy or diabetic kidney disease" as the search term, through searching transient triple differential (TTD) (https://db.idrblab.org/ttd/, version date is 20 Jun 2019) (25), DRUGBANK (https://www.drugbank.ca/, version date is 2017 Nov 8) (26), and DisGeNET

(http://www.disenet.org/web/disgenet/menu/home, version: 6.0) (27).

1.5 Gene information standardization

The obtained targets information of drugs and diseases were normalization in UniProt database (https://www.uniprot.org/, 2019) (28), which was a protein database with the most abundant information and resources. Finally, the network of "drug-target-disease" was constructed with Cytoscape

(http://cytoscapeweb.cytoscape.org/, version: 3.2.1) (29).

1.6 Signal pathway analysis

The predicted targets gene was analyzed by protein interaction in STRING database (https://string-db.org/)(30), and the target pathway was analyzed by ClueGO (http://www.cytoscape.org/apps/cleego) (31).

\section{Results}

2.1 Results of prescription containing AM reducing urinary albumin excretion rate

7 high-quality randomized controlled trials (RCTs) were included in the treatment of DN with prescription containing AM. The basic information included the author (date of publication), research methods, diagnostic criteria (diabetes, DN), intervention drugs, composition of prescription containing AM, duration of intervention, number of patients included and results of urinary protein excretion rate (Table 1). The results of one study was not included in the statistics because of the inconsistent result, which showed that prescription containing AM could effectively reduce the urinary protein excretion rate(32). The total results showed that prescription containing AM could significantly reduce the urinary protein excretion rate [95\% MD-43.30 (- 57.00, - 29.61)] (Fig. 2). 
Table 1

basic information included in the study

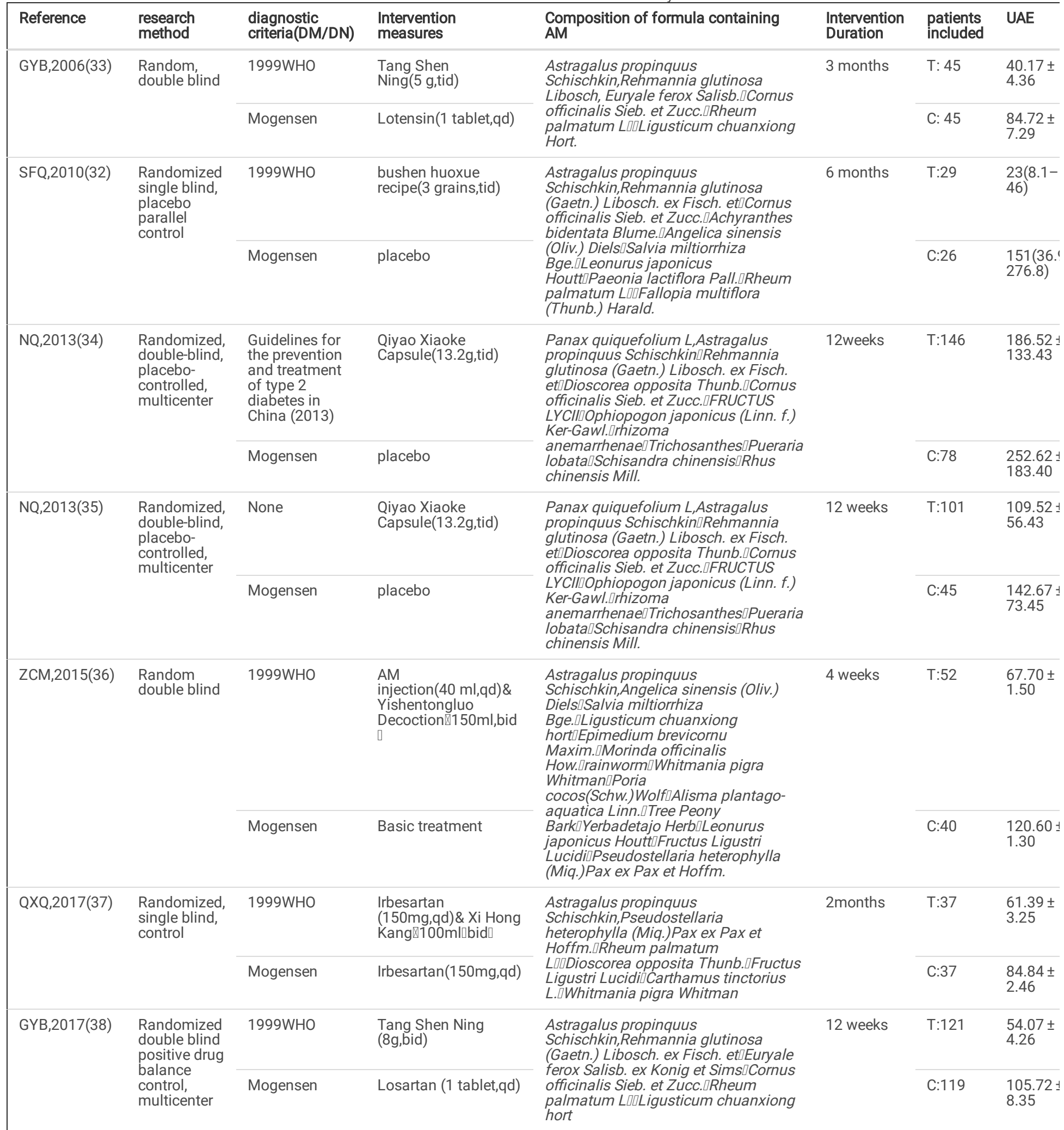

Abbreviation:1999WHO: Diagnostic criteria for diabetes established by the World Health Organization in 1999; Urinary protein excretion rate:UAE.

\subsection{Active ingredients of AM}

A total of 51 active components of AM were retrieved, including Adenine, Calycosin, Astragaloside VII, and the SMILES of active components was recorded (Table 2). 


\begin{tabular}{|c|c|c|}
\hline Number & Ingredient name & SMILES \\
\hline 1 & adenine & $\mathrm{C} 1=\mathrm{NC} 2=\mathrm{NC}=\mathrm{NC}(=\mathrm{C} 2 \mathrm{~N} 1) \mathrm{N}$ \\
\hline 2 & calycosin & $\mathrm{COC} 1=\mathrm{C}(\mathrm{C}=\mathrm{C}(\mathrm{C}=\mathrm{C} 1) \mathrm{C} 2=\mathrm{COC} 3=\mathrm{C}(\mathrm{C} 2=0) \mathrm{C}=\mathrm{CC}(=\mathrm{C} 3) 0) \mathrm{O}$ \\
\hline 3 & astragaloside vii & $\begin{array}{l}\mathrm{CC} 1(\mathrm{C}(\mathrm{CCC} 23 \mathrm{C} 1 \mathrm{C}(\mathrm{CC} 4 \mathrm{C} 2(\mathrm{C} 3) \mathrm{CCC} 5(\mathrm{C} 4(\mathrm{CC}(\mathrm{C5C} 6(\mathrm{CCC}(\mathrm{O} 6) \mathrm{C}(\mathrm{C}) \\
(\mathrm{C}) 0 \mathrm{CC} 7 \mathrm{C}(\mathrm{C}(\mathrm{C}(\mathrm{C}(07) \mathrm{CO}) 0) 0) 0) \mathrm{C}) 0) \mathrm{C}) \mathrm{C}) \mathrm{OC} 8 \mathrm{C}(\mathrm{C}(\mathrm{C}(\mathrm{C}(\mathrm{O} 8) \mathrm{CO}) 0) 0) 0 \mathrm{OC9C}(\mathrm{C}(\mathrm{C}(\mathrm{CO9}) 0) 0) 0) \mathrm{C}\end{array}$ \\
\hline 4 & astragaloside $v$ & 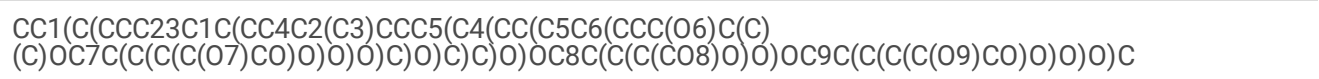 \\
\hline 5 & astragaloside vi & $\begin{array}{l}\mathrm{CC} 1(\mathrm{C}(\mathrm{CCC} 23 \mathrm{C} 1 \mathrm{C}(\mathrm{CC} 4 \mathrm{C} 2(\mathrm{C} 3) \mathrm{CCC} 5(\mathrm{C} 4(\mathrm{CC}(\mathrm{C5C} 6(\mathrm{CCC}(\mathrm{O} 6) \mathrm{C}(\mathrm{C}) \\
(\mathrm{C}) 0) \mathrm{C}) 0) \mathrm{C}) \mathrm{C}) 0 \mathrm{OC} 7 \mathrm{C}(\mathrm{C}(\mathrm{C}(\mathrm{C}(\mathrm{O} 7) \mathrm{CO}) 0) 0) 0) 0 \mathrm{C} 8 \mathrm{C}(\mathrm{C}(\mathrm{C}(\mathrm{CO} 8) 0) 0) 0 \mathrm{C}(\mathrm{C}(\mathrm{C}(\mathrm{C}(09) \mathrm{CO}) 0) 0) 0) \mathrm{C}\end{array}$ \\
\hline 6 & astramembrannin i & $\begin{array}{l}\mathrm{CC} 1(\mathrm{C}(\mathrm{CCC} 23 \mathrm{C} 1 \mathrm{C}(\mathrm{CC} 4 \mathrm{C} 2(\mathrm{C} 3) \mathrm{CCC} 5(\mathrm{C} 4(\mathrm{CC}(\mathrm{C} 5 \mathrm{C} 6(\mathrm{CCC}(\mathrm{O} 6) \mathrm{C}(\mathrm{C}) \\
(\mathrm{C}) \mathrm{O}) \mathrm{C}) 0) \mathrm{C}) \mathrm{C}) \mathrm{OC} 7 \mathrm{C}(\mathrm{C}(\mathrm{C}(\mathrm{C}(\mathrm{O}) \mathrm{CO}) 0) 0) 0) \mathrm{OC} 8 \mathrm{C}(\mathrm{C}(\mathrm{C}(\mathrm{CO} 8) 0) 0) \mathrm{C}\end{array}$ \\
\hline 7 & astragaloside ii & 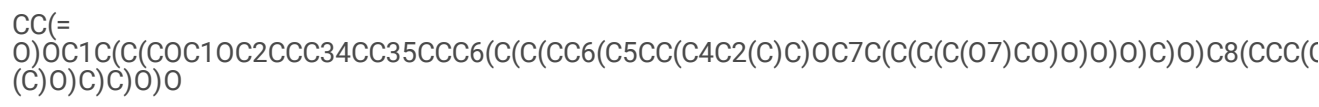 \\
\hline 8 & astragaloside i & 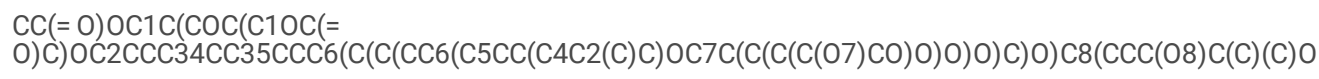 \\
\hline 9 & astragaloside iv & 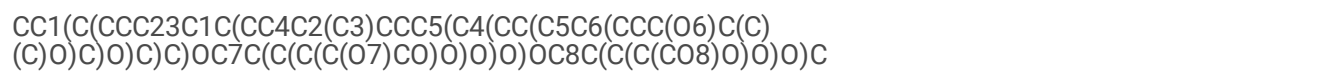 \\
\hline 10 & astragaloside iii & $\begin{array}{l}\mathrm{CC} 1(\mathrm{C}(\mathrm{CCC} 23 \mathrm{C} 1 \mathrm{C}(\mathrm{CC} 4 \mathrm{C} 2(\mathrm{C} 3) \mathrm{CCC} 5(\mathrm{C} 4(\mathrm{CC}(\mathrm{C} 5 \mathrm{C} 6(\mathrm{CCC}(\mathrm{O} 6) \mathrm{C}(\mathrm{C}) \\
(\mathrm{C}) \mathrm{O}) \mathrm{C}) 0) \mathrm{C}) \mathrm{C}) 0) 0 \mathrm{C} 7 \mathrm{C}(\mathrm{C}(\mathrm{C}(\mathrm{CO} 7) 0) 0) 0 \mathrm{C} 8 \mathrm{C}(\mathrm{C}(\mathrm{C}(\mathrm{C}(\mathrm{O}) \mathrm{CO}) 0) 0) 0) \mathrm{C}\end{array}$ \\
\hline 11 & canavanine & $C(C O N=C(N) N) C(C(=0) O) N$ \\
\hline 12 & chrysanthemaxanthin & $\mathrm{CC} 1=\mathrm{CC}(\mathrm{CC}(\mathrm{C} 1 \mathrm{C}=\mathrm{CC}(=\mathrm{CC}=\mathrm{CC}(=\mathrm{CC}=\mathrm{CC}=\mathrm{C}(\mathrm{C}) \mathrm{C}=\mathrm{CC}=\mathrm{C}(\mathrm{C}) \mathrm{C} 2 \mathrm{C}=\mathrm{C} 3 \mathrm{C}(\mathrm{CC}(\mathrm{CC} 3(\mathrm{O} 2) \mathrm{C}) \mathrm{O})(\mathrm{C}) \mathrm{C}) \mathrm{C}) \mathrm{C})(\mathrm{C}) \mathrm{C}) \mathrm{O}$ \\
\hline 13 & cycloastragenol & $\operatorname{cc} 1(\mathrm{C}(\mathrm{CCC} 23 \mathrm{C} 1 \mathrm{C}(\mathrm{CC} 4 \mathrm{C} 2(\mathrm{C} 3) \mathrm{Ccc} 5(\mathrm{C} 4(\mathrm{CC}(\mathrm{C} 5 \mathrm{C} 6(\mathrm{CcC}(\mathrm{O} 6) \mathrm{C}(\mathrm{C})(\mathrm{C}) 0) \mathrm{C}) 0) \mathrm{C}) \mathrm{C}) 0) 0) \mathrm{C}$ \\
\hline 14 & choline & $\mathrm{C}[\mathrm{N}+](\mathrm{C})(\mathrm{C}) \mathrm{CCO}$ \\
\hline 15 & 3,5-dimethoxystilbene & $\mathrm{COC} 1=\mathrm{CC}(=\mathrm{CC}(=\mathrm{C} 1) \mathrm{C}=\mathrm{CC} 2=\mathrm{CC}=\mathrm{CC}=\mathrm{C} 2) \mathrm{OC}$ \\
\hline 16 & formononetin & $\mathrm{COC} 1=\mathrm{CC}=\mathrm{C}(\mathrm{C}=\mathrm{C} 1) \mathrm{C} 2=\mathrm{COC} 3=\mathrm{C}(\mathrm{C} 2=0) \mathrm{C}=\mathrm{CC}(=\mathrm{C} 3) \mathrm{O}$ \\
\hline 17 & foliosidine & $\mathrm{CC}(\mathrm{C})(\mathrm{C}(\mathrm{COC} 1=\mathrm{CC}=\mathrm{CC} 2=\mathrm{C} 1 \mathrm{~N}(\mathrm{C}(=\mathrm{O}) \mathrm{C}=\mathrm{C} 2 \mathrm{OC}) \mathrm{C}) 0) \mathrm{O}$ \\
\hline 18 & guanosine & $\mathrm{C} 1=\mathrm{NC} 2=\mathrm{C}(\mathrm{N} 1 \mathrm{C} 3 \mathrm{C}(\mathrm{C}(\mathrm{C}(\mathrm{O} 3) \mathrm{CO}) 0) 0) \mathrm{N}=\mathrm{C}(\mathrm{NC2}=0) \mathrm{N}$ \\
\hline 19 & 20-hexadecanoylingenol & $\operatorname{cccccccccccccccc}(=0) 0 \operatorname{co} 1=\operatorname{cc} 2 \mathrm{C} 3 \mathrm{C}(\mathrm{C} 3(\mathrm{C}) \mathrm{C}) \operatorname{cc}(\mathrm{C} 4(\mathrm{C} 2=0) \mathrm{C}=\mathrm{C}(\mathrm{C}(\mathrm{C} 4(\mathrm{C} 10) 0) 0) \mathrm{C}) \mathrm{C}$ \\
\hline 20 & 2-hydroxy-3-methoxystrychnine & $\mathrm{COC} 1=\mathrm{C}(\mathrm{C}=\mathrm{C} 2 \mathrm{C}(=\mathrm{C} 1) \mathrm{N} 3 \mathrm{C} 4 \mathrm{C} 25 \mathrm{CCN} 6 \mathrm{C} 5 \mathrm{CC} 7 \mathrm{C} 4 \mathrm{C}(\mathrm{CC} 3=0) \mathrm{OCC}=\mathrm{C} 7 \mathrm{C} 6) \mathrm{O}$ \\
\hline 21 & lupeol & 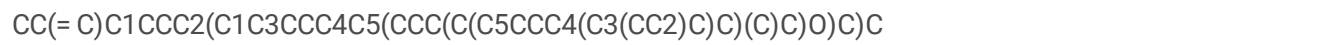 \\
\hline 22 & isorhamnetin & $\mathrm{COC} 1=\mathrm{C}(\mathrm{C}=\mathrm{CC}(=\mathrm{C} 1) \mathrm{C} 2=\mathrm{C}(\mathrm{C}(=\mathrm{O}) \mathrm{C} 3=\mathrm{C}(\mathrm{C}=\mathrm{C}(\mathrm{C}=\mathrm{C} 302) 0) 0) 0) 0$ \\
\hline 23 & kumatakenin & $\mathrm{COC} 1=\mathrm{CC}(=\mathrm{C} 2 \mathrm{C}(=\mathrm{C} 1) \mathrm{OC}(=\mathrm{C}(\mathrm{C} 2=0) \mathrm{OC}) \mathrm{C} 3=\mathrm{CC}=\mathrm{C}(\mathrm{C}=\mathrm{C} 3) 0) \mathrm{O}$ \\
\hline 24 & kaempferol & $\mathrm{C} 1=\mathrm{CC}(=\mathrm{CC}=\mathrm{C} 1 \mathrm{C} 2=\mathrm{C}(\mathrm{C}(=0) \mathrm{C} 3=\mathrm{C}(\mathrm{C}=\mathrm{C}(\mathrm{C}=\mathrm{C} 302) 0) 0) 0) 0$ \\
\hline 25 & rhamnocitrin & $\mathrm{COC} 1=\mathrm{CC}(=\mathrm{C} 2 \mathrm{C}(=\mathrm{C} 1) \mathrm{OC}(=\mathrm{C}(\mathrm{C} 2=0) 0) \mathrm{C} 3=\mathrm{CC}=\mathrm{C}(\mathrm{C}=\mathrm{C} 3) 0) \mathrm{O}$ \\
\hline 26 & quercetin & $\mathrm{C} 1=\mathrm{CC}(=\mathrm{C}(\mathrm{C}=\mathrm{C} 1 \mathrm{C} 2=\mathrm{C}(\mathrm{C}(=0) \mathrm{C} 3=\mathrm{C}(\mathrm{C}=\mathrm{C}(\mathrm{C}=\mathrm{C} 302) 0) 0) 0) 0) 0$ \\
\hline 27 & soyasapogenol b & $\operatorname{cc} 1(\operatorname{Cc} 2 \mathrm{C} 3=\operatorname{ccc} 4 \mathrm{C} 5(\operatorname{Ccc}(\mathrm{C}(\mathrm{C} 5 \mathrm{CCC} 4(\mathrm{C} 3(\mathrm{Ccc} 2(\mathrm{C}(\mathrm{C} 1) \mathrm{O}) \mathrm{C}) \mathrm{C}) \mathrm{C})(\mathrm{C}) \mathrm{CO}) 0) \mathrm{C}) \mathrm{C}$ \\
\hline 28 & sucrose & $\mathrm{C}(\mathrm{C} 1 \mathrm{C}(\mathrm{C}(\mathrm{C}(\mathrm{C}(\mathrm{O} 1) \mathrm{OC} 2(\mathrm{C}(\mathrm{C}(\mathrm{C}(\mathrm{O} 2) \mathrm{CO}) 0) 0) \mathrm{CO}) 0) 0) 0) 0$ \\
\hline 29 & suffruticoside a & $\mathrm{CC}(=0) \mathrm{C} 1=\mathrm{C}(\mathrm{C}=\mathrm{C}(\mathrm{C}=\mathrm{C} 1) 0 \mathrm{C}) 0 \mathrm{C} 2 \mathrm{C}(\mathrm{C}(\mathrm{C}(\mathrm{C}(\mathrm{O} 2) \operatorname{coc} 3 \mathrm{C}(\mathrm{C}(\mathrm{CO} 3)(\mathrm{COC}(=0) \mathrm{C} 4=\mathrm{CC}(=\mathrm{C}(\mathrm{C}(=\mathrm{C} 4) 0) 0) 0) 0) 0) 0)$ \\
\hline 30 & uridine & $\mathrm{C} 1=\mathrm{CN}(\mathrm{C}(=0) \mathrm{NC} 1=0) \mathrm{C} 2 \mathrm{C}(\mathrm{C}(\mathrm{C}(\mathrm{O} 2) \mathrm{CO}) 0) 0$ \\
\hline 31 & betaine & $\mathrm{C}[\mathrm{N}+](\mathrm{C})(\mathrm{C}) \mathrm{CC}(=0)[\mathrm{O}-]$ \\
\hline 32 & beta-sitosterol & $\operatorname{ccc}(\operatorname{ccc}(\mathrm{C}) \mathrm{C} 1 \mathrm{CcC} 2 \mathrm{C} 1(\mathrm{Ccc} 3 \mathrm{C} 2 \mathrm{CC}=\mathrm{C} 4 \mathrm{C} 3(\operatorname{ccc}(\mathrm{C} 4) \mathrm{O}) \mathrm{C}) \mathrm{C}) \mathrm{C}(\mathrm{C}) \mathrm{C}$ \\
\hline 33 & acetic acid & $\mathrm{CC}(=0) 0$ \\
\hline 34 & gamma-sitosterol & $\operatorname{ccC}(\operatorname{CcC}(\mathrm{C}) \mathrm{C} 1 \mathrm{CCC} 2 \mathrm{C} 1(\mathrm{CCC} 3 \mathrm{C} 2 \mathrm{CC}=\mathrm{C} 4 \mathrm{C} 3(\mathrm{CCC}(\mathrm{C} 4) 0) \mathrm{C}) \mathrm{C}) \mathrm{C}(\mathrm{C}) \mathrm{C}$ \\
\hline 35 & folinic acid & $\mathrm{C} 1 \mathrm{C}(\mathrm{N}(\mathrm{C} 2=\mathrm{C}(\mathrm{N} 1) \mathrm{N}=\mathrm{C}(\mathrm{NC2}=0) \mathrm{N}) \mathrm{C}=0) \mathrm{CNC3}=\mathrm{CC}=\mathrm{C}(\mathrm{C}=\mathrm{C} 3) \mathrm{C}(=0) \mathrm{NC}(\mathrm{CCC}(=0) 0) \mathrm{C}(=0) 0$ \\
\hline 36 & Mairin & $\mathrm{C} 1 \mathrm{C}(\mathrm{C}(=0) \mathrm{C} 2=\mathrm{CC}=\mathrm{CC}=\mathrm{C} 201) \mathrm{C} 3=\mathrm{CC}=\mathrm{CC}=\mathrm{C} 3$ \\
\hline 37 & Jaranol & $\mathrm{COC} 1=\mathrm{CC}(=\mathrm{C} 2 \mathrm{C}(=\mathrm{C} 1) \mathrm{OC}(=\mathrm{C}(\mathrm{C} 2=0) \mathrm{OC}) \mathrm{C} 3=\mathrm{CC}=\mathrm{C}(\mathrm{C}=\mathrm{C} 3) 0) \mathrm{O}$ \\
\hline
\end{tabular}




\begin{tabular}{|c|c|c|}
\hline Number & Ingredient name & SMILES \\
\hline 38 & hederagenin & $\operatorname{cc} 1(\operatorname{Ccc} 2(\operatorname{ccc} 3(C(=\operatorname{ccc} 4 \mathrm{C} 3(\operatorname{ccc} 5 \operatorname{C} 4(\operatorname{CcC}(\operatorname{C5}(\mathrm{C}) \operatorname{co}) 0) \mathrm{C}) \mathrm{C}) \mathrm{C} 2 \mathrm{C} 1) \mathrm{C}) \mathrm{C}(=0) 0) \mathrm{C}$ \\
\hline 39 & $\begin{array}{l}\text { (3S,8S,9S,10R,13R,14S,17R)-10,13- } \\
\text { dimethyl-17-[(2R,5S)-5-propan-2- } \\
\text { yloctan-2- } \\
\text { yl]-2,3,4,7,8,9,11,12,14,15,16,17- } \\
\text { dodecahydro-1H- } \\
\text { cyclopenta[a]phenanthren-3-ol }\end{array}$ & $\operatorname{cccc}(\operatorname{ccc}(\mathrm{C}) \mathrm{C} 1 \mathrm{CcC} 2 \mathrm{C} 1(\mathrm{ccc} 3 \mathrm{C} 2 \mathrm{CC}=\mathrm{C} 4 \mathrm{C} 3(\operatorname{ccc}(\mathrm{C} 4) \mathrm{O}) \mathrm{C}) \mathrm{C}) \mathrm{C}(\mathrm{C}) \mathrm{C}$ \\
\hline 40 & 3,9-di-0-methylnissolin & $\mathrm{COC} 1=\mathrm{CC} 2=\mathrm{C}(\mathrm{C}=\mathrm{C} 1) \mathrm{C} 3 \mathrm{C}(\mathrm{CO} 2) \mathrm{C} 4=\mathrm{C}(\mathrm{O} 3) \mathrm{C}(=\mathrm{C}(\mathrm{C}=\mathrm{C} 4) \mathrm{OC}) \mathrm{OC}$ \\
\hline 41 & $\begin{array}{l}\text { 5'-hydroxyiso-muronulatol-2',5'-di- } \\
\text { O-glucoside }\end{array}$ & $\mathrm{c} 1(0) \mathrm{ccc} 2 \mathrm{c}(\mathrm{OCC}(\mathrm{c} 3 \mathrm{c}(\mathrm{OC} 4 \mathrm{OC}(\mathrm{CO}) \mathrm{C}(0) \mathrm{C}(0) \mathrm{C} 40) \mathrm{c}(\mathrm{OC}) \mathrm{c}(\mathrm{OC}) \mathrm{c}(\mathrm{OC} 5 \mathrm{OC}(\mathrm{CO}) \mathrm{C}(0) \mathrm{C}(0) \mathrm{C} 50) \mathrm{c} 3) \mathrm{c} 2) \mathrm{c} 1$ \\
\hline 42 & 7-0-methylisomucronulatol & $\mathrm{COC} 1=\mathrm{CC} 2=\mathrm{C}(\mathrm{CC}(\mathrm{CO} 2) \mathrm{C} 3=\mathrm{C}(\mathrm{C}(=\mathrm{C}(\mathrm{C}=\mathrm{C} 3) \mathrm{OC}) \mathrm{OC}) 0) \mathrm{C}=\mathrm{C} 1$ \\
\hline 43 & $\begin{array}{l}\text { 9,10-dimethoxypterocarpan-3-0- } \beta \text { - } \\
\text { D-glucoside }\end{array}$ & $\mathrm{O}(\mathrm{c} 4 \mathrm{cc} 3 \mathrm{c}(\mathrm{C} 2 \mathrm{C}(\mathrm{c} 5 \mathrm{c}(\mathrm{O} 2) \mathrm{c}(\mathrm{OC}) \mathrm{c}(\mathrm{OC}) \mathrm{cc} 5) \mathrm{CO} 3) \mathrm{cc} 4) \mathrm{C} 1 \mathrm{C}(\mathrm{C}(\mathrm{C}(\mathrm{C}(\mathrm{CO}) 01) 0) 0) 0$ \\
\hline 44 & $\begin{array}{l}\text { (6aR,11aR)-9,10-dimethoxy- } \\
\text { 6a,11a-dihydro-6H- } \\
\text { benzofurano[3,2-c]chromen-3-ol }\end{array}$ & $\mathrm{COC} 1=\mathrm{C}(\mathrm{C} 2=\mathrm{C}(\mathrm{C}=\mathrm{C} 1) \mathrm{C} 3 \mathrm{COC} 4=\mathrm{C}(\mathrm{C} 302) \mathrm{C}=\mathrm{CC}(=\mathrm{C} 4) 0) \mathrm{OC}$ \\
\hline 45 & Bifendate & $\mathrm{COC} 1=\mathrm{C} 2 \mathrm{C}(=\mathrm{C}(\mathrm{C}(=\mathrm{C} 1) \mathrm{C}(=0) \mathrm{OC}) \mathrm{C} 3=\mathrm{C} 4 \mathrm{C}(=\mathrm{C}(\mathrm{C}=\mathrm{C} 3 \mathrm{C}(=0) 0 \mathrm{C}) \mathrm{OC}) 0 \mathrm{CO} 4) 0 \mathrm{CO} 2$ \\
\hline 46 & isoflavanone & $\mathrm{C} 1 \mathrm{C}(\mathrm{C}(=0) \mathrm{C} 2=\mathrm{CC}=\mathrm{CC}=\mathrm{C} 2 \mathrm{O} 1) \mathrm{C} 3=\mathrm{CC}=\mathrm{CC}=\mathrm{C} 3$ \\
\hline 47 & FA & $\mathrm{C} 1=\mathrm{CC}(=\mathrm{CC}=\mathrm{C} 1 \mathrm{C}(=0) \mathrm{NC}(\mathrm{CCC}(=0) 0) \mathrm{C}(=0) 0) \mathrm{NCC} 2=\mathrm{CN}=\mathrm{C} 3 \mathrm{C}(=\mathrm{N} 2) \mathrm{C}(=0) \mathrm{N}=\mathrm{C}(\mathrm{N} 3) \mathrm{N}$ \\
\hline 48 & $\begin{array}{l}\text { (3R)-3-(2-hydroxy-3,4- } \\
\text { dimethoxyphenyl)chroman-7-ol }\end{array}$ & $\mathrm{COC} 1=\mathrm{C}(\mathrm{C}(=\mathrm{C}(\mathrm{C}=\mathrm{C} 1) \mathrm{C} 2 \mathrm{CC} 3=\mathrm{C}(\mathrm{C}=\mathrm{C}(\mathrm{C}=\mathrm{C} 3) 0) \mathrm{OC} 2) \mathrm{O}) \mathrm{OC}$ \\
\hline 49 & $\begin{array}{l}\text { isomucronulatol-7,2'-di-0- } \\
\text { glucosiole }\end{array}$ & $\mathrm{Coc} 1=\mathrm{C}(\mathrm{C}(=\mathrm{C}(\mathrm{C}=\mathrm{C} 1) \mathrm{C} 2 \mathrm{CC} 3=\mathrm{C}(\mathrm{C}=\mathrm{C}(\mathrm{C}=\mathrm{C} 3) 0 \mathrm{C} 4 \mathrm{C}(\mathrm{C}(\mathrm{C}(\mathrm{C}(\mathrm{O} 4) \mathrm{CO}) 0) 0) 0) 0 \mathrm{C} 2) 0 \mathrm{C} 5 \mathrm{C}(\mathrm{C}(\mathrm{C}(\mathrm{C}(05) \mathrm{CO}) 0) 0) 0)$ \\
\hline 50 & $\begin{array}{l}\text { 1,7-Dihydroxy-3,9-dimethoxy } \\
\text { pterocarpene }\end{array}$ & $\mathrm{COC} 1=\mathrm{CC}(=\mathrm{C} 2 \mathrm{C}(=\mathrm{C} 1) \mathrm{OCC} 3=\mathrm{C} 2 \mathrm{OC} 4=\mathrm{CC}(=\mathrm{CC}(=\mathrm{C} 34) 0) \mathrm{OC}) \mathrm{O}$ \\
\hline 51 & Soyasaponin 1 & $\begin{array}{l}\mathrm{CC} 1 \mathrm{C}(\mathrm{C}(\mathrm{C}(\mathrm{C}(01) \mathrm{OC} 2 \mathrm{C}(\mathrm{C}(\mathrm{C}(\mathrm{OC} 2 \mathrm{OC} 3 \mathrm{C}(\mathrm{C}(\mathrm{C}(\mathrm{OC} 30 \mathrm{C} 4 \mathrm{CCC} 5(\mathrm{C}(\mathrm{C} 4(\mathrm{C}) \mathrm{CO}) \mathrm{CCC} 6(\mathrm{C} 5 \mathrm{CC}=\mathrm{C} 7 \mathrm{C} 6(\mathrm{CCC} 8(\mathrm{C} 7 \mathrm{CC}(\mathrm{CC} 80 \\
(\mathrm{C}) \mathrm{C}) \mathrm{C}) \mathrm{C}) \mathrm{C}) \mathrm{C}) \mathrm{C}(=0) 0) 0) 0) \mathrm{CO}) 0) 0) 0) 0) 0\end{array}$ \\
\hline
\end{tabular}

2.3 Verification and prediction targets of AM

According to the screening criteria, 396 verification targets, and 2330 prediction targets were searched in the Swiss target prediction database.

2.4 DN targets

According to the screening criteria, 120 targets, including Angiotensin-converting enzyme (ACE), Methylenetetrahydrofolate reductase (MTHFR), Solute carrier family 15 member 1 (SLC15A1), Solute carrier family 15 member 2 (SLC15A2), were searched in the databases of Drugbank, TTD, and DisGeNET.

\subsection{Disease-Target-Drug Intersection}

The effective composition, targets of DN and AM were imported into Cytoscape, and the network diagram of "drug-component-target-disease" was drawn (Fig. 3), which intuitively reflected the relationship among disease, drug and targets. There were 76 nodes and 165 lines in the diagram. Among them, the purple triangle represented the common targets, the blue triangle represented the effective components of AM, and the DN and AM were respectively represented by white rectangles. The results showed that there were 21 targets, including ACE, Vascular endothelial growth factor A (VEGFA), Janus Kinase 1 (JAK1), V-jun avian sarcoma virus 17 oncogene homolog (JUN), Serine/threonine-protein kinase (SGK1), Matrix metalloproteinase-9 (MMP9), Matrix metalloproteinase-1 (MMP1), Matrix metalloproteinase-2 (MMP2), hydrolase RelA (RELA), Endothelin-1 receptor (EDNRA), Rho-associated protein kinase 1 (ROCK1), Heparanase (HPSE), Peptidyl-cysteine S-nitrosylase (NOS2), Type-1 angiotensin II receptor (AGTR1), Vitamin D3 receptor (VDR), Broad substrate specificity ATP-binding cassette transporter (ABCG2), Serum paraoxonase/arylesterase 1 (PON1), Peroxisome proliferator-activated receptor gamma (PPARG), Interleukin-6 (IL6), Cytochrome P450 3A4 (CYP3A4), Nuclear factor, erythroid derived 2, like 2 (NFE2L2). (Table 3) 
Table 3

Basic information of Targets(Uniprot database)

\begin{tabular}{|c|c|c|c|c|}
\hline Entry & Entry name & Protein names & Gene names & Length \\
\hline P12821 & ACE_HUMAN & Angiotensin-converting enzyme & ACE & 1306 \\
\hline P15692 & VEGFA_HUMAN & Vascular endothelial growth factor $A$ & VEGFA & 232 \\
\hline P23458 & JAK1_HUMAN & Tyrosine-protein kinase & JAK1 & 1154 \\
\hline P05412 & JUN_HUMAN & Proto-oncogene c-Jun & JUN & 331 \\
\hline 000141 & SGK1_HUMAN & Serine/threonine-protein kinase & SGK1 & 431 \\
\hline P14780 & MMP9_HUMAN & Matrix metalloproteinase-9 & MMP9 & 707 \\
\hline P03956 & MMP1_HUMAN & Matrix metalloproteinase-1 & MMP1 & 469 \\
\hline P08253 & MMP2_HUMAN & Matrix metalloproteinase-2 & MMP2 & 660 \\
\hline Q04206 & TF65_HUMAN & Transcription factor p65 & RELA & 551 \\
\hline P25101 & EDNRA_HUMAN & Endothelin receptor type $A$ & EDNRA & 427 \\
\hline Q13464 & ROCK1_HUMAN & Rho-associated, coiled-coil-containing protein kinase I & ROCK1 & 1354 \\
\hline Q9Y251 & HPSE_HUMAN & Heparanase & HPSE & 543 \\
\hline P35228 & NOS2_HUMAN & Peptidyl-cysteine S-nitrosylase NOS2 & NOS2 & 1153 \\
\hline P30556 & AGTR1_HUMAN & Type-1 angiotensin II receptor & AGTR1 & 359 \\
\hline P11473 & VDR_HUMAN & Vitamin D3 receptor & VDR & 427 \\
\hline Q9UNQ0 & ABCG2_HUMAN & Broad substrate specificity ATP-binding cassette transporter & ABCG2 & 655 \\
\hline P27169 & PON1_HUMAN & Serum paraoxonase/arylesterase 1 & PON1 & 355 \\
\hline P37231 & PPARG_HUMAN & Peroxisome proliferator-activated receptor gamma & PPARG & 505 \\
\hline P05231 & IL6_HUMAN & Interleukin-6 & IL6 & 212 \\
\hline P08684 & CP3A4_HUMAN & Cytochrome P450 3A4 & CYP3A4 & 503 \\
\hline Q16236 & NF2L2_HUMAN & Nuclear factor, erythroid derived 2, like 2 & NFE2L2 & 605 \\
\hline
\end{tabular}

2.3 Protein Interaction

The STRING database was used to construct the interaction between proteins, in which the red line represented the evidence of fusion, the green line represented the evidence of proximity, the blue line represented the evidence of coexistence, the purple line represented the experimental evidence, the yellow line represented the evidence of text mining, the light blue line represented the evidence of database, and the black line represented the evidence of co expression. (Fig. 4)

2.6 Signal pathway enrichment results

ClueGO was used to analyze the signal pathway enrichment of common targets of AM and DN, the results showed that the signal pathways such as interleukin-4 and 13 signaling, activation of matrix metalloproteases, ephrin signaling are most related to AM treatment of DN (Fig. 5). Among them, AM was most likely to play a therapeutic role by acting on Interleukin-4 (IL-4) / Interleukin-13 (IL-13), Matrix metalloproteinase (MMPs), autoantigen / Tumor necrosis factor - a (TNF - a), collagen degradation, c-Jun N-terminal kinase (JNK) and p-38 mitogen-activated protein kinase (p38MAPK) pathway (Fig. 6).

Abbreviation:IL-22: Interleukin-22; IL-6: Interleukin-6; IL-23: Interleukin-23; STAT3: signal transducer and activator of transcription 3; HIF-a: Hypoxia inducible factor alpha; IL-17: Interleukin-17; IL-4: Interleukin-4; IL-13: Interleukin-13; TNFR1: Recombinant Human Tumor Necrosis Factor Receptor Type 1; TAK1: Transforming growth factor kinase 1; IKK: inhibitor of nuclear factor kappa-B kinase; NFKB1: Nuclear Factor Kappa B Subunit 1; AP-1: activator protein 1; MMPs: matrix metalloproteinase; TNFa: Tumor Necrosis Factora; SASP: senes-cence-associated secretory phenotype; TGF- $\beta$ : transforming growth factor- $\beta$; JNK:C-Jun N-terminal kinase; p38MAPK: p38 mitogen-activated protein kinase.

\section{Discussion}

Through clinical observation, we found that large dose of AM could significantly reduce the urinary protein of early stage DN patients. Through searching the relevant database, we screened high-quality clinical studies and finally included 7 RCTs that meet the inclusion criteria. The results showed that prescription containing AM could effectively reduce the urinary protein excretion rate of DN patients [95\% MD-43.30 (-57.00, - 29.61)], indicating that prescription containing AM could be used as an alternative treatment for DN. Finally, 51 effective components of AM, 396 verified targets and 2330 predicted targets were found. A total of $120 \mathrm{DN}$ related targets were retrieved. 21 targets were found to be shared by DN and AM with Cytoscape. The related targets mainly involved in interleukin-4 and 13 signaling, activation of matrix meta Lloproteinases, ephrin signaling and other signaling pathways. Therefore, AM may play a therapeutic role through these targets, which providing an alternative treatment for DN. 
For interleukin-4 and 13 signaling, IL-4 and IL-13 receptors were expressed in human cells, and constantly changeed in the course of disease, which was related to cell inflammation and cell proliferation (39). For activation of matrix metalloproteases, MMP was involved from embryonic development to apoptosis of cells, which was closely related to inflammation and atherosclerosis. The MMP modulator could be used as a preventive treatment for individuals with risk of atherosclerosis and vascular weakening (40). Eph / ephrin signaling was a two-way signaling pathway, which include in developmental and homeostatic environments. The interaction between Eph / ephrin signaling and cells and tissues in the urinary system was also important in the damage response of podocytes and glomeruli (41). These studies showed that AM may play a therapeutic role by regulating chronic inflammation of DN, improving microvascular circulation and maintaining cell function.

At present, there is no study to predict the possible targest of AM for DN through network pharmacology. A pharmacological study showed that AM could reduce proteinuria, reverse glomerular filtration and improve early or other stages of DN in streptozotocin induced diabetic animal models, which may be related down regulating the expression of TGF- $\beta 1$, Smad2/3 and a-SMA through affecting TGF- $\beta$ /SMADs signal pathway, delaying renal fibrosis in diabetic mice (16). In clinical practice and statistics of clinical research, we found that prescription containing AM could significantly reduce the urinary protein excretion rate of DN patients. Based on the theory of system biology, we applied the network analysis of biological system, and with more comprehensive potential mechanism, such as AM may act on IL-4 / IL-13, MMPs, autoantigen/TNF-a, collagen degradation, JNK and p38MAPK pathway to treat DN, which provides guidance for the later research direction.

Only high-quality clinical studies were selected in the included our study, which may lead to publication bias. At the same time, the results of AM and DN targets were seached from the currently recorded data in the database, which may also lead to publication bias. At the same time, animal experiments and in vitro studies are still lacking.

\section{Conclusion}

Prescription containing AM could effectively reduce the urinary protein excretion rate of DN patients, which may be used as alternative treatment for DN, and its mechanism may includ IL-4 / IL-13, MMPs, autoantigen/TNF-a, collagen degradation, JNK and p38MAPK through the signal pathways of interleukin-4 and 13 signaling, activation of matrix metalloproteinases, ephrin signaling, which would be verify through animal experiments or vitro experiments later.

\section{Abbreviations}

AM: Astragalus membranaceus; DN: diabetic nephropathy; TCMSP: Traditional Chinese Medicine systems taxonomy database and analysis platform; TCMID; Traditional Chinese medicine integrated database; BATMAN-TCM; Bioinformatics analysis tool for molecular mechanism of traditio Internal Chinese medicine; HIT: health information technology; SMILES: Simplified molecular input line entry specification; TTD: transient triple differential; ACE: Angiotensin-converting enzyme; VEGFA: Vascular endothelial growth factor A; JAK1: Janus Kinase 1; IL-4: interleukin-4; IL-13: interleukin-13; TGF- $\beta$; transforming growth factor- $\beta$; SMADs: drosophila mothers against decapentaplegic protein; RCTs: randomized controlled trials; WHO: World Health Organization; MTHFR: Methylenetetrahydrofolate reductase; SLC15A1: Solute carrier family 15 member 1; SLC15A2: Solute carrier family 15 member 2; VEGFA: Vascular endothelial growth factor A; JAK1: Janus Kinase 1; JUN: V-jun avian sarcoma virus 17 oncogene homolog; SGK1: Serine/threonine-protein kinase; MMP9: Matrix metalloproteinase-9; MMP1: Matrix metalloproteinase-1; MMP2: Matrix metalloproteinase-2; RELA: hydrolase RelA; EDNRA: Endothelin-1 receptor; ROCK1: Rhoassociated protein kinase 1; HPSE: Heparanase; NOS2: Peptidyl-cysteine S-nitrosylase; AGTR1: Type-1 angiotensin II receptor; VDR: Vitamin D3 receptor; ABCG2: Broad substrate specificity ATP-binding cassette transporter; PON1: Serum paraoxonase/arylesterase 1; PPARG: Peroxisome proliferator-activated receptor gamma; IL6: Interleukin-6; CYP3A4: Cytochrome P450 3A4; NFE2L2: Nuclear factor, erythroid derived 2, like 2; MMPs: Matrix metalloproteinase; TNF a: Tumor necrosis factor - a; JNK: c-Jun N-terminal kinase; p38MAPK: p-38 mitogen-activated protein kinase; IL-22: Interleukin-22; IL-23: Interleukin-23; STAT3: signal transducer and activator of transcription 3; HIF-a: Hypoxia inducible factor alpha; IL-17: Interleukin-17; IL-13: Interleukin-13; TNFR1: Recombinant Human Tumor Necrosis Factor Receptor Type 1; IKK: inhibitor of nuclear factor kappa-B kinase; NFKB1: Nuclear Factor Kappa B Subunit 1; AP-1: activator protein 1; SASP: senes-cence-associated secretory phenotype; TGF- $\beta$ : transforming growth factor- $\beta$.

\section{Declarations}

\section{Ethics approval and consent to participate}

Not Applicable

\section{Consent for publication}

Not Applicable

\section{Availability of data and materials}

The data used to support the results of this study can be obtained from the corresponding author.

\section{Author contributions}

FML designed the protocol. XDA, LYD, DJ, RRZ, and YYD carried out the active ingredient, and relevant targets search. FML and XDA contributed to data extraction and results analysis. YRZ and SHZ corrected the related data. All authors approved the final version of the manuscript.

\section{Competing Interests}


Not Applicable

\section{Funding section}

This work was supported by the National Public Welfare Industry Special (201507001-11), National Traditional Chinese Medicine Administration of Traditional Chinese Medicine Science and Technology Research Project (2016ZX03). The funders had no role in the study design, data collection, data analysis, interpretation, or writing of the report.

\section{Acknowledgements}

Not Applicable

\section{References}

1. Reddy MA, Tak Park J, Natarajan R. Epigenetic modifications in the pathogenesis of diabetic nephropathy. Semin Nephrol. 2013;33(4):341-53.

2. Tuttle KR, Bakris GL, Bilous RW, Chiang JL, de Boer IH, Goldstein-Fuchs J, et al. Diabetic kidney disease: a report from an ADA Consensus Conference. Am J Kidney Dis. 2014;64(4):510 - 33.

3. Reidy K, Kang HM, Hostetter T, Susztak K. Molecular mechanisms of diabetic kidney disease. J Clin Invest. 2014;124(6):2333-40.

4. Dai H, Liu Q, Liu B. Research Progress on Mechanism of Podocyte Depletion in Diabetic Nephropathy. J Diabetes Res. 2017;2017:2615286.

5. Szrejder M, Piwkowska A. AMPK signalling: Implications for podocyte biology in diabetic nephropathy. Biol Cell. 2019;111(5):109-20.

6. Roman-Ramos R, Almanza-Perez JC, Fortis-Barrera A, Angeles-Mejia S, Banderas-Dorantes TR, Zamilpa-Alvarez A, et al. Antioxidant and anti-inflammatory effects of a hypoglycemic fraction from Cucurbita ficifolia Bouche in streptozotocin-induced diabetes mice. Am J Chin Med. 2012;40(1):97-110.

7. Anzaldua DA, Schmitz PG. Aliskiren as an alternative in a patient with life-threatening ACE inhibitor-induced angioedema. Am J Kidney Dis. 2008;51(3):532-3.

8. Groop PH, Cooper ME, Perkovic V, Emser A, Woerle HJ, von Eynatten M. Linagliptin lowers albuminuria on top of recommended standard treatment in patients with type 2 diabetes and renal dysfunction. Diabetes Care. 2013;36(11):3460-8.

9. Kim MJ, Frankel AH, Donaldson M, Darch SJ, Pusey CD, Hill PD, et al. Oral cholecalciferol decreases albuminuria and urinary TGF-beta1 in patients with type 2 diabetic nephropathy on established renin-angiotensin-aldosterone system inhibition. Kidney Int. 2011;80(8):851-60.

10. Wang H, Zhou Q, Tong X. Tong XiaoLin'Experience of Astragalus membranaceus in the treatment of diabetic complications. Global traditional Chinese Medicine. 2013;6(04):272-4.

11. Li Q, Zhang HM, Fei YT. [Treatment of diabetic nephropathy by integrative medicine: a multi-center prospective cohort study]. Zhongguo Zhong Xi Yi Jie He Za Zhi. 2012;32(3):317-21.

12. Li M, Wang W, Xue J, Gu Y, Lin S. Meta-analysis of the clinical value of Astragalus membranaceus in diabetic nephropathy. J Ethnopharmacol. 2011;133(2):412-9.

13. Zhang J, Xie X, Li C, Fu P. Systematic review of the renal protective effect of Astragalus membranaceus (root) on diabetic nephropathy in animal models. J Ethnopharmacol. 2009;126(2):189-96.

14. Wang ZS, Xiong F, Xie XH, Chen D, Pan JH, Cheng L. Astragaloside IV attenuates proteinuria in streptozotocin-induced diabetic nephropathy via the inhibition of endoplasmic reticulum stress. BMC Nephrol. 2015;16:44.

15. Liao H, Hu L, Cheng X, Wang X, Li J, Banbury L, et al. Are the Therapeutic Effects of Huangqi (Astragalus membranaceus) on Diabetic Nephropathy Correlated with Its Regulation of Macrophage iNOS Activity? J Immunol Res. 2017;2017:3780572.

16. Wang Y, Lin C, Ren Q, Liu Y, Yang X. Astragaloside effect on TGF-beta1, SMAD2/3, and alpha-SMA expression in the kidney tissues of diabetic KKAy mice. Int J Clin Exp Pathol. 2015;8(6):6828-34.

17. Hopkins AL. Network pharmacology. Nat Biotechnol. 2007;25(10):1110-1.

18. Ru J, Li P, Wang J, Zhou W, Li B, Huang C, et al. TCMSP: a database of systems pharmacology for drug discovery from herbal medicines. J Cheminform. 2014;6:13.

19. Xue R, Fang Z, Zhang M, Yi Z, Wen C, Shi T. TCMID: Traditional Chinese Medicine integrative database for herb molecular mechanism analysis. Nucleic Acids Res. 2013;41(Database issue):D1089-95.

20. Liu Z, Guo F, Wang Y, Li C, Zhang X, Li H, et al. BATMAN-TCM: a Bioinformatics Analysis Tool for Molecular mechANism of Traditional Chinese Medicine. Sci Rep. 2016;6:21146.

21. Wang Y, Bryant SH, Cheng T, Wang J, Gindulyte A, Shoemaker BA, et al. PubChem BioAssay: 2017 update. Nucleic Acids Res. 2017;45(D1):D955-d63.

22. Ohno-Machado L. Using health information technology for clinical decision support and predictive analytics. J Am Med Inform Assoc. 2017;24(1):1.

23. Gfeller D, Grosdidier A, Wirth M, Daina A, Michielin O, Zoete V. SwissTargetPrediction: a web server for target prediction of bioactive small molecules. Nucleic Acids Res. 2014;42(Web Server issue):W32-8.

24. Tetko IV, Tanchuk VY. Application of associative neural networks for prediction of lipophilicity in ALOGPS 2.1 program. J Chem Inf Comput Sci. 2002;42(5):1136-45.

25. Chen X, Ji ZL, Chen YZ. TTD: Therapeutic Target Database. Nucleic Acids Res. 2002;30(1):412-5. 
26. Wishart DS, Knox C, Guo AC, Cheng D, Shrivastava S, Tzur D, et al. DrugBank: a knowledgebase for drugs, drug actions and drug targets. Nucleic Acids Res. 2008;36(Database issue):D901-6.

27. Pinero J, Bravo A, Queralt-Rosinach N, Gutierrez-Sacristan A, Deu-Pons J, Centeno E, et al. DisGeNET: a comprehensive platform integrating information on human disease-associated genes and variants. Nucleic Acids Res. 2017;45(D1):D833-d9.

28. Reorganizing the protein space at the Universal Protein Resource (UniProt). Nucleic Acids Res. 2012;40(Database issue):D71-5.

29. Shannon P, Markiel A, Ozier O, Baliga NS, Wang JT, Ramage D, et al. Cytoscape: a software environment for integrated models of biomolecular interaction networks. Genome Res. 2003;13(11):2498-504.

30. von Mering C, Huynen M, Jaeggi D, Schmidt S, Bork P, Snel B. STRING: a database of predicted functional associations between proteins. Nucleic Acids Res. 2003;31(1):258-61.

31. Bindea G, Mlecnik B, Hackl H, Charoentong P, Tosolini M, Kirilovsky A, et al. ClueGO: a Cytoscape plug-in to decipher functionally grouped gene ontology and pathway annotation networks. Bioinformatics. 2009;25(8):1091-3.

32. Si F, Zhou S, Cui Y, Zhang X, Zhang Z. The intervention effect of Bushen Huoxue Method on urinary albumin excretion rate and hemodynamics in diabetic nephropathy. Liaoning Journal of traditional Chinese Medicine. 2010;37(06):979-80.

33. Gao Y, Zhao H, Guan S, Zhou H, Geng J, Xie P, et al. Clinical study on Tangshenning in the treatment of early diabetic nephropathy with deficiency of Qi and Yin and stagnation of collaterals and blood stasis. Chinese Journal of traditional Chinese Medicine. 2006(07):409-11.

34. Ni Q, Jiang S, Xiao Y, Feng X, Li J, Zhu Z, et al. Clinical observation on 146 cases of diabetic nephropathy treated with Qiyao Xiaoke Capsule and Western Medicine. Journal of traditional Chinese Medicine. 2013;54(06):484-7.

35. Ni Q, Jiang S, Xiao Y, Feng X, Li J, Zhu Z, et al. Multicenter, randomized, double-blind, placebo-controlled clinical observation on the treatment of early diabetic nephropathy with Qiyao Xiaoke Capsule. Chinese Journal of traditional Chinese Medicine. 2013;28(08):2479-82.

36. Zheng C, Bai Z, Li Y, Xu S, Ban W, Zhang H, et al. Treatment of diabetic nephropathy with Yishentongluo Decoction. Chinese Journal of experimental prescriptions. 2015;21(09):175-9.

37. Qu X. Clinical study on xihongkang in the treatment of early diabetic nephropathy [master]: xinjiang medical university; 2017.

38. Gao Y, Zhou H, Guan S, Zhao J, Li B, Chen L, et al. Multi center randomized double blind controlled clinical trial of Tangshenning granule in the treatment of diabetic nephropathy. Chinese Journal of traditional Chinese Medicine. 2017;32(11):5212-5.

39. McCormick SM, Heller NM. Commentary. IL-4 and IL-13 receptors and signaling. Cytokine. 2015;75(1):38-50.

40. Mittal R, Patel AP, Debs LH, Nguyen D, Patel K, Grati M, et al. Intricate Functions of Matrix Metalloproteinases in Physiological and Pathological Conditions. J Cell Physiol. 2016;231(12):2599-621.

41. Weiss AC, Kispert A. Eph/ephrin signaling in the kidney and lower urinary tract. Pediatr Nephrol. 2016;31(3):359-71.

\section{Figures}




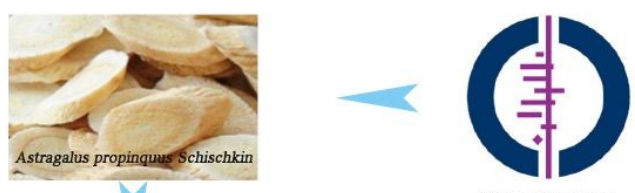

High-quality RCTs
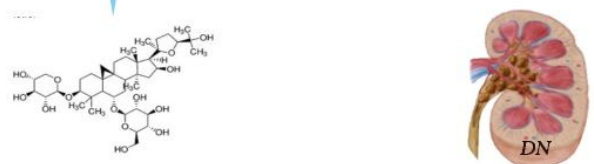

TCMSP、TCMID、BATMAN

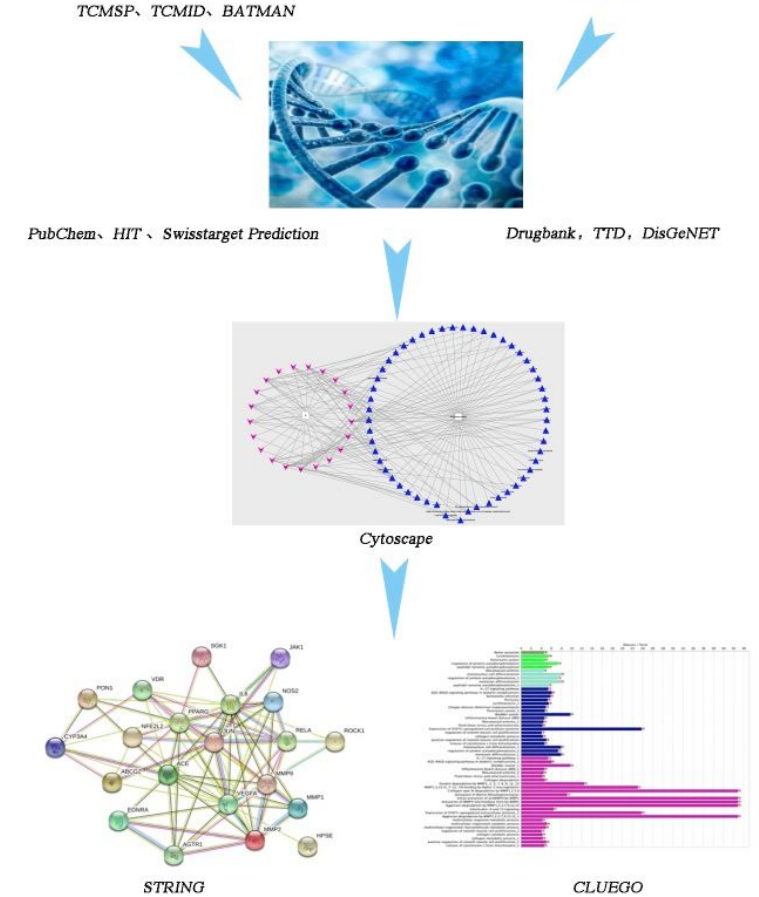

Figure 1

Flow chart

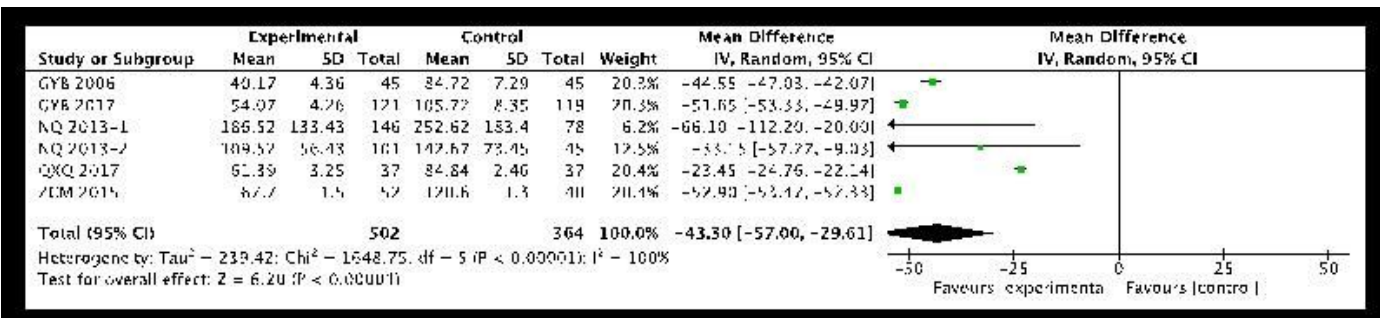

Figure 2

urinary protein excretion rate 


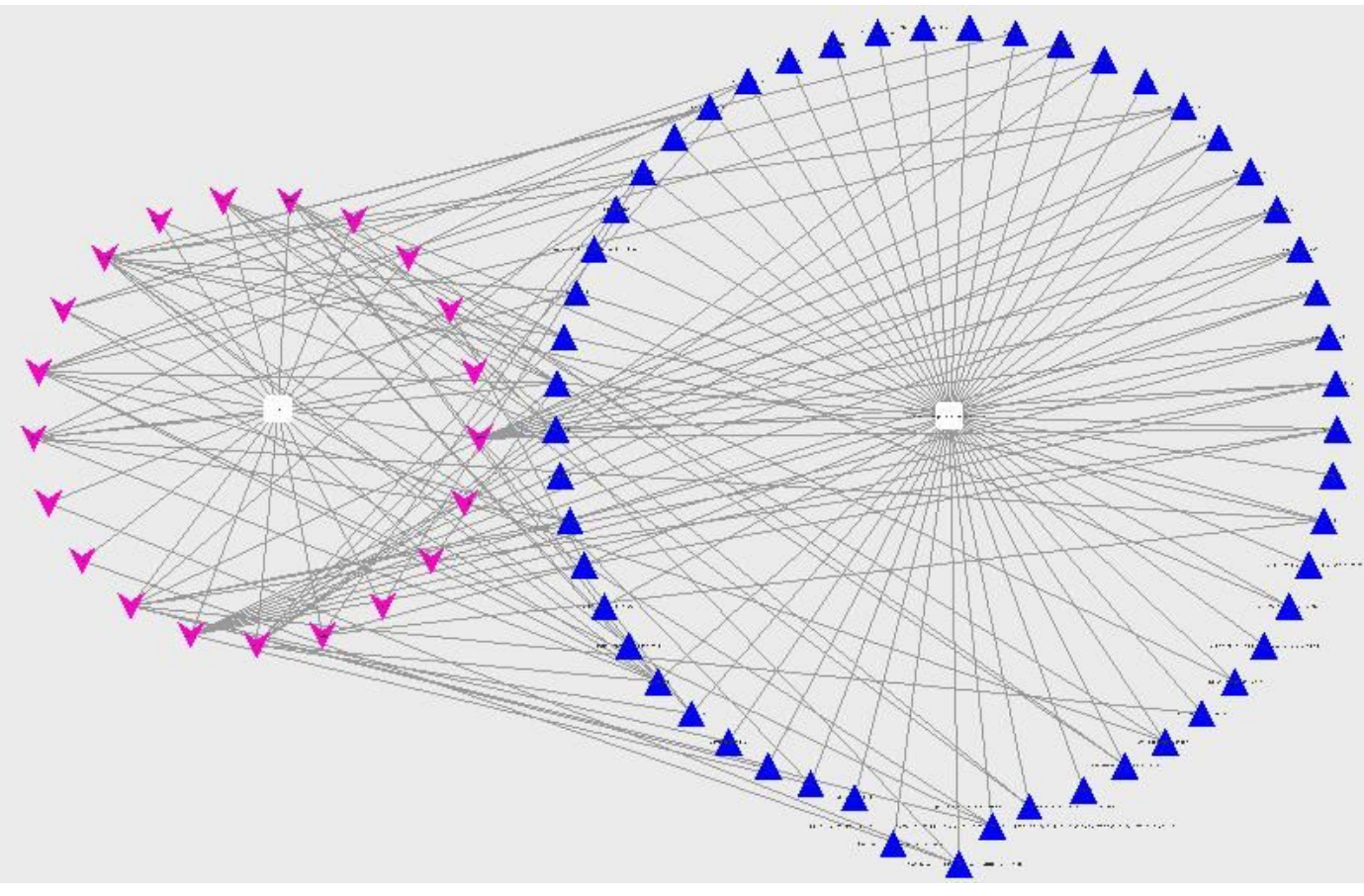

Figure 3

Drug-Component-Validation/Prediction Target-Disease network

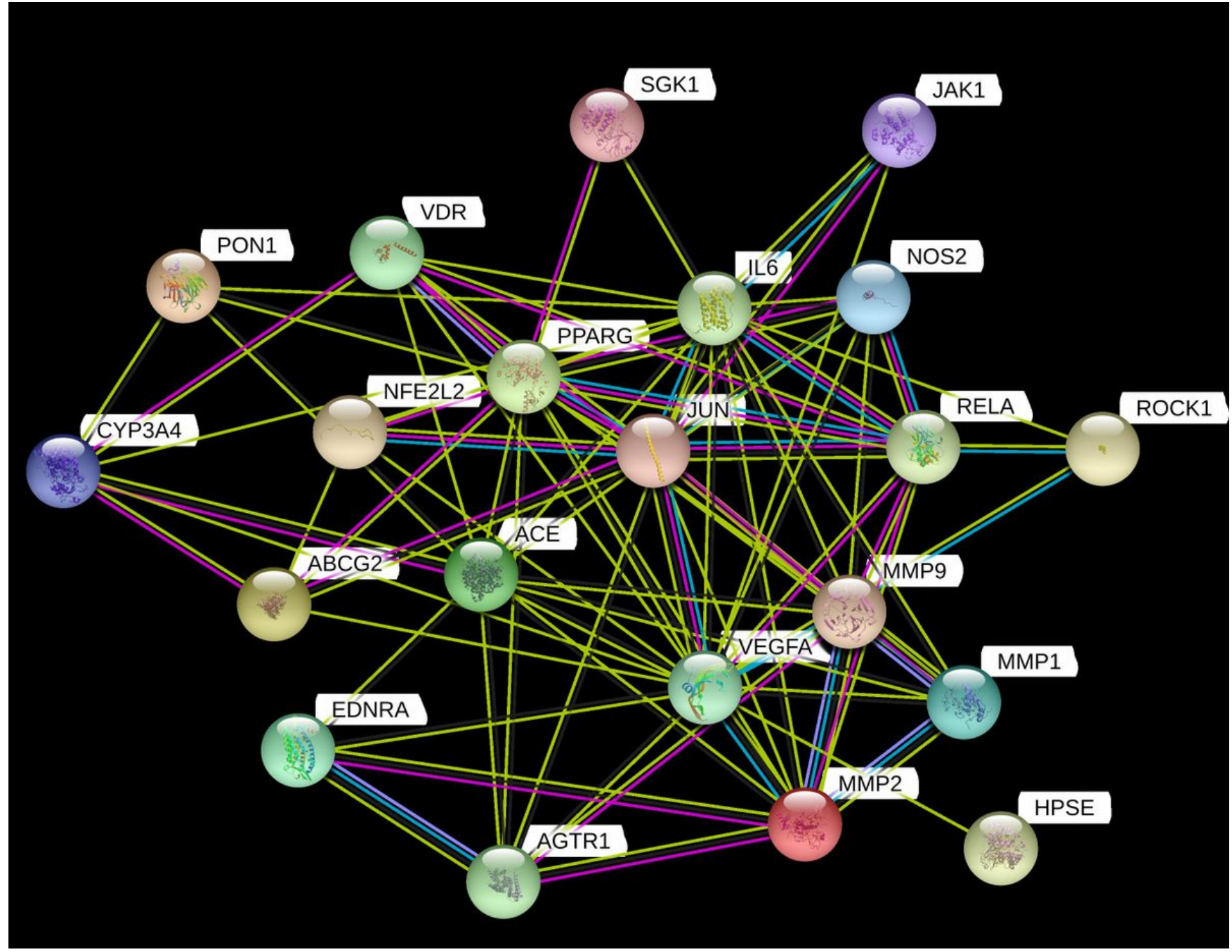

Figure 4 
Protein interaction based on STRING
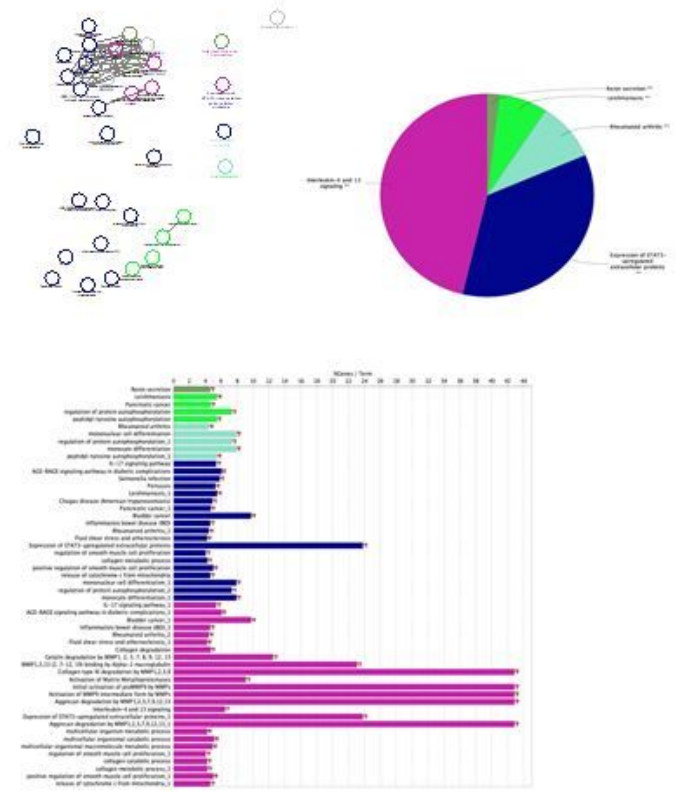

\section{Figure 5}

Signal pathway enrichment

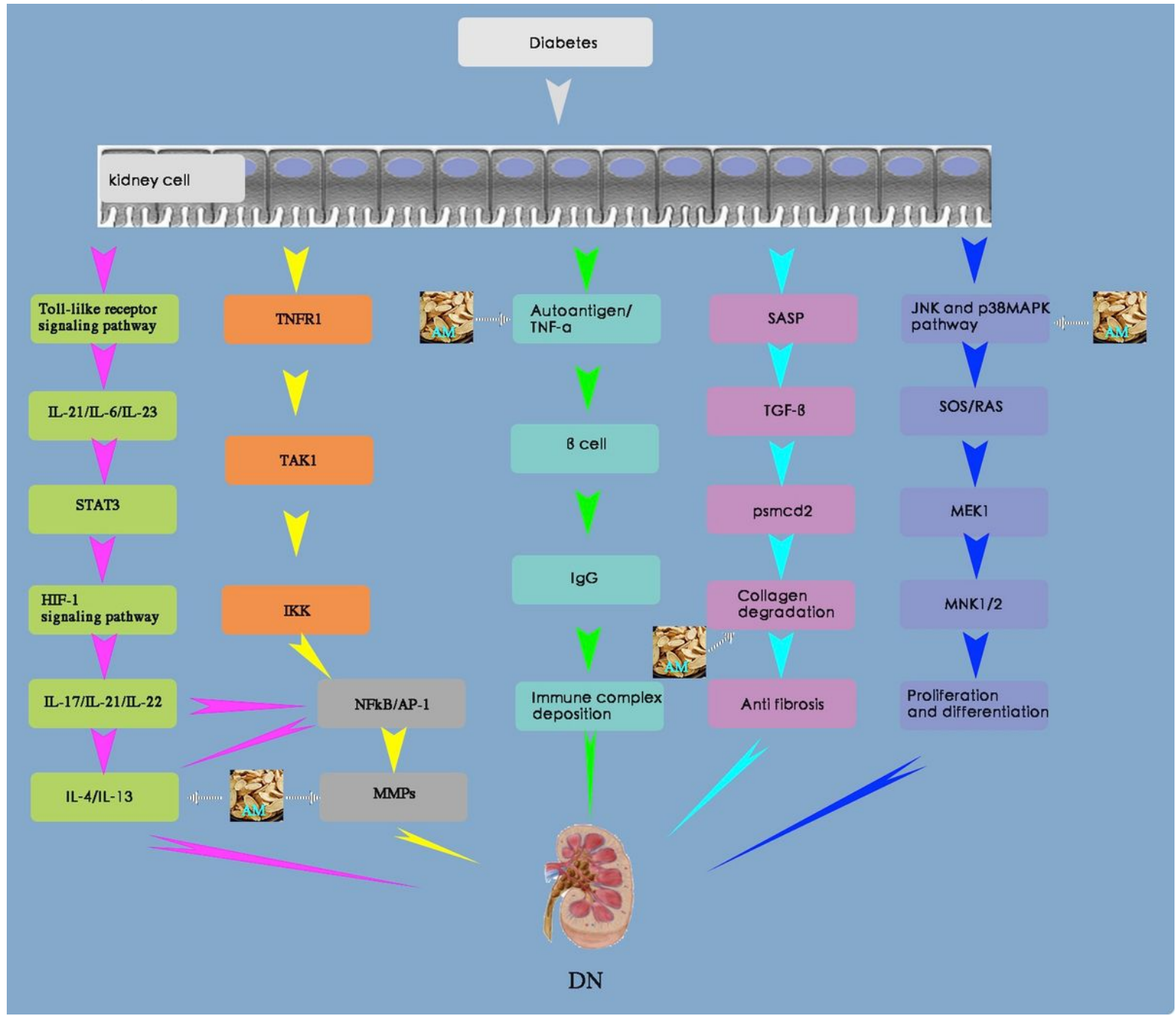

Figure 6 
The potential mechanism of AM in the treatment of DN

Page 14/14 